\title{
Extracellular vesicles-transmitted miR-21a-5p altered microglia polarization after hypoxia- ischemic injury in neonatal mice via STAT3 pathways
}

\section{Danqing Xin}

Shandong University

Yijing Zhao

Shandong University

Tingting Li

Shandong University

Hongfei Ke

Shandong University

Chengcheng Gai

Shandong University

\section{Xiaofan Guo}

Loma Linda University

\section{Wenqiang Chen}

Shandong University

Dexiang Liu

Shandong University

Wang Zhen ( $\nabla$ wangzhen@sdu.edu.cn )

Shandong University School of Medicine https://orcid.org/0000-0003-3173-6961

\section{Research}

Keywords: extracellular vesicles, STAT3, microglia, miR-21a-5p, hypoxia-ischemia

Posted Date: March 22nd, 2021

DOl: https://doi.org/10.21203/rs.3.rs-313905/v1

License: (c) (1) This work is licensed under a Creative Commons Attribution 4.0 International License. Read Full License 


\section{Abstract}

Background We previously reported that mesenchymal stromal cells (MSCs)-derived extracellular vesicles (EVs) exhibit protective effects in hypoxia-ischemia (HI) brain damage. The neuroprotective action was connected with its anti-inflammatory effect. However, the mechanisms involved with this effect have not been determined.

Methods A modified version of the Rice-Vannucci method was performed on postnatal day 7 mouse pups to induce neonatal HI brain injury. The model of oxygen-glucose deprivation (OGD) was established in BV2 cells to mimic $\mathrm{HI}$ injury in vitro. Mice or BV-2 cells received EVs and EVs-miR-21a ${ }^{\text {inhibitor }}$ at indicative time post-injury. In vivo, brain water content and TTC staining were used to evaluate the effects of EVs on $\mathrm{HI}$ brain injury. Immunofluorescence staining was used to observe the effect of EVs on the polarization of microglia. The effect of EVs on p-STAT3 was assessed by Western blot. In vitro, the effect of EVs on cell survival was evaluated by CCK8. Expression of miR-21a-5p and inflammatory factors was measured using qRT-PCR. Dual-Luciferase Reporter Assay was performed to illustrate the link between miR-21a-5p and STAT3. The role of miR-21a-5p in EVs on HI injury and ODG injury was further investigated by using EVs-miR-21a $a^{\text {inhibitor. }}$

Results By using OGD mimicking HI injury in vitro, we found that MSCs-EVs treatment elevated cell viability following OGD exposure in BV-2 cells. MSCs-EVs treatment impeded microglia-mediated neuroinflammation, shifted microglia toward M2 polarization, and suppressed the phosphorylation of selective signal transducer and activator of transcription 3 (STAT3) in microglia after $\mathrm{HI}$ exposure in vitro and in vivo. In light of miR-21a-5p being the most highly expressed miRNA in MSCs-EVs interacting with the STAT3 pathway, further work focused on this pathway. Notably, MSCs-EVs treatment increased HIreduced miR-21a-5p levels in BV-2 cells. Diminishing miR-21a-5p in MSCs-EVs partially attenuated its effect on microglia polarization and STAT3 phosphorylation following $\mathrm{HI}$ exposure in vitro and in vivo.

Conclusions Our study suggested that MSCs-EVs attenuated HI brain damage in neonatal mice via shuttling miR-21a-5p, which induced microglia M2 polarization by targeting STAT3.

\section{Introduction}

Neonatal hypoxia-ischemia (HI) brain damage is one of the major causes of death and/or long-term neurodevelopmental disabilities [1]. Evidence suggests that neuroinflammation via releasing proinflammatory mediators lead to the alteration of neuronal functions and contribute to persistent $\mathrm{HI}$ brain damage [2, 3]. As the main effectors of neuroinflammation following $\mathrm{HI}$ insult, microglia can be polarized into proinflammatory M1 or anti-inflammatory M2 [4]. The balance between the M1 phenotype and the $\mathrm{M} 2$ phenotype plays an important role in regulating neuroinflammation and brain homeostasis.

Mesenchymal stromal cells (MSCs) possess broad immunoregulatory properties and could modulate inflammation-related diseases[5]. For example, human umbilical cord-derived MSCs exerted anti-diabetic effects and alleviated islet dysfunction in type 2 diabetic mice by switching macrophages from M1 
phenotype to M2 phenotype [6]. Evidence suggests the immunomodulatory properties of MSCs related to its paracrine effect [7]. For example, stimulation of MSCs with lipopolysaccharide (LPS) can secrete certain factors to influence macrophage function $[5,8]$. Recently, extracellular vesicles (EVs), recognized as an important MSCs paracrine factor, contribute to the positive effects of MSCs [9]. EVs were classified into small EVs (50-100 nm), medium EVs $(100 \mathrm{~nm}-1 \mu \mathrm{m})$ and large EVs $(1 \mu \mathrm{m}-5 \mu \mathrm{m})$ according to their size or density [10]. MSCs-EVs may promote an immunosuppressive response through the induction of immature dendritic cell, switch macrophages from M1 toward M2 phenotype, secreting anti-inflammatory cytokines[9]. The latest research shows that MSCs-EVs play important roles in ischemic brain damage. For instance, MSCs-EVs ameliorated ischemia-induced hippocampal synaptic damage in adult mice [11], improved the neurodevelopmental outcomes following $\mathrm{HI}$ injury of the newborn animals $[12,13]$. Human Wharton's jelly MSCs-derived EVs prevented neuronal cells against oxygen-glucose deprivation (OGD)induced apoptosis [14]. Moreover, MSCs-EVs suppressed LPS-initiated inflammation in BV-2 cells [15]. Within our own laboratory, we have found that MSCs-EVs administration exerts the neuroprotective and anti-inflammatory effect on $\mathrm{HI}$ injury in neonatal mice $[16,17]$. However, its underlying mechanism is still unknown.

In the present study, we used $\mathrm{HI}$ injury in neonatal mice in vivo and oxygen-glucose deprivation (OGD) mimicking $\mathrm{HI}$ injury in vitro. We found that miR-21a-5p in MSCs-EVs has dramatic effects on the regulation of microglia polarization to resolve neuroinflammation following $\mathrm{HI}$ insult by targeting selective signal transducer and activator of transcription 3 (STAT3).

\section{Materials And Method}

\section{Materials}

Reagents and primer sequences are listed in Table S1 and Table S2 of supplementary material respectively.

\section{$\mathrm{HI}$ model and treatments}

The $\mathrm{HI}$ model was induced on postnatal day 7 (P7) according to the methods of Rice et al, as described in our previous publication [17]. Briefly, C57BL/6J male mouse pups (P7) were anesthetized with $2 \%$ isoflurane and the right common carotid artery was separated and ligated. After skin suturing and disinfection, the pups were put back to their dam for $60 \mathrm{~min}$, and then exposed in a hypoxia chamber (humidified at $8 \% \mathrm{O}_{2}+92 \% \mathrm{~N}_{2}$ ) at $37^{\circ} \mathrm{C}$ for $90 \mathrm{~min}$. Finally, the mice were put back to the dam to continue feeding for the follow-up experiment. In the Sham operation group mice, only the common carotid artery was exposed without ligation and hypoxia treatment.

The mice were randomly divided into the following groups: Sham + vehicle (PBS) group, $\mathrm{HI}+$ vehicle (PBS) group, $\mathrm{HI}+$ EVs group, $\mathrm{HI}+$ EVs-miR-21a ${ }^{\mathrm{INC}}$ group and $\mathrm{HI}+\mathrm{EVs}-\mathrm{miR}-21 \mathrm{a}^{\text {inhibitor }}$ group. A total of 100 $\mu \mathrm{g}$ EVs dissolved in $50 \mu \mathrm{L}$ PBS was administered via an intracardial injection at $24 \mathrm{~h}$ after $\mathrm{HI}$ insult for one time [17]. 


\section{Culture and identification of MSCs}

MSCs from bone marrow were harvested from C57BL/6J mice (4 weeks old) as previously described and modified [18]. Briefly, the cells were washed out from the femur and tibia of the donor mice with a syringe with 20 needles, filtered through $70 \mu \mathrm{m}$ filter, centrifuged at $350 \times \mathrm{g}$ for $5 \mathrm{~min}$, and then suspended in the complete medium (DME/F-12 containing 10\% fetal bovine serum (FBS), $2 \mathrm{mM} \mathrm{L-glutamine,} 100 \mathrm{U} / \mathrm{mL}$ penicillin and $100 \mu \mathrm{g} / \mathrm{mL}$ streptomycin), and cultured in $5 \% \mathrm{CO}_{2}$ air environment at $37^{\circ} \mathrm{C}$. After $3 \mathrm{~h}$, the fresh complete medium was replaced to remove the non-adherent cells. The medium was replaced every $8 \mathrm{~h}$ in the next $24 \mathrm{~h}$, and then every $3 \mathrm{~d}$. Adherent cells (passage 0 ) reached approximately $90 \%$ confluence in $14 \mathrm{~d}$ and were lifted by incubating in $0.25 \%$ trypsin/ $1 \mathrm{mM}$ ethylenediaminetetraacetic acid. Lifted cells were cultured in $5 \% \mathrm{CO}_{2}$ air environment at $37^{\circ} \mathrm{C}$, and the complete medium was replaced every $3 \mathrm{~d}$. The spindle-shaped morphology MSCs at passage 5-8 were used for subsequent experiments.

Alizarin Red and Oil Red 0 staining were utilized to identify osteogenic and adipogenic differentiation of MSCs, respectively. Flow Cytometric Analysis (FACS) was used to detect the surface markers of MSCs. MSCs were incubated with the following antibodies: CD29-FITC, CD44 -PE/Cy7, Sca-1-APC, CD11b-FITC, CD45-APC, CD31-FITC, CD117-APC, CD14-FITC, CD19-PE, Ly6G-PerCP and CD4-FITC. The result was acquired with FACS flow cytometer $\mathrm{C6}$.

\section{Harvest and identification of MSCs-EVs and contents analysis}

As previously described, the total EVs isolation kit (qEV, iZonScience, \#1001622) was used for isolation and identification of EVs [17]. In short, when MSCs were fused to about $60 \%$, the medium was replaced by EVs-free serum medium for $24 \mathrm{~h}$, then collect the medium and centrifuge at $5752 \times \mathrm{g}$ for 30 min to obtain the supernatant and filter $(0.22 \mu \mathrm{m})$. The supernatant (about $15 \mathrm{~mL}$ ) was transferred to a $100000 \mathrm{MWCO}$ ultrafiltration tube and centrifuged at $4000 \times \mathrm{g}$ for $30 \mathrm{~min}$. The sample in each ultrafiltration tube was concentrated to about 200-300 $\mu \mathrm{L}$ and transferred to the qEV column. The remaining steps are carried out according to the manufacturer's instructions. Finally, $3 \mathrm{~mL}$ of PBS suspension containing EVs was used immediately or stored at $-80^{\circ} \mathrm{C}$.

Western blot was used to measure the common markers of EVs, such as CD9 and TSG-101 [10]. The morphology of EVs was observed with use of TEM (HITACHI Limited, Japan). The qNano platform (Izon Sciences Ltd, NZ) was used to

determine the size and concentration of EVs.

\section{MSCs-EVs labeled with PKH67}

In order to examine the distribution of EVs, the green fluorescent dye PKH67 (Sigma-Aldrich Co., St Louis, MO, USA) was used to mark the EVs with according to the manufacturers' direction. Briefly, PKH67 dye (4 $\mu \mathrm{L})$ was mixed into Diluent $\mathrm{C}(1 \mathrm{~mL})$ to obtain the PKH67 solution. Then, PKH67 solution $(1 \mathrm{~mL})$ and diluent EVs (1 mL) were combined within centrifugation tube for $5 \mathrm{~min}, 2 \mathrm{~mL} 1 \%$ bovine serum albumin 
(BSA) was added to centrifugation tube to stop dyeing. Then, the mixture was ultracentrifuged (100000 $\times$ g) for 70 min to obtain EVs precipitate, followed by washing again with PBS $(100000 \times \mathrm{g})$ for $70 \mathrm{~min}$. Finally, PKH67-labeled EVs were resuspended in PBS.

\section{Tracking the distribution and location of MSCs-EVs}

In order to determine the distribution and localization of EVs in BV-2 cells, cells seeded in 24-well plates were treated with OGD and then incubated with PKH67-labeled EVs $(10 \mu \mathrm{g} / \mathrm{mL})$ for $24 \mathrm{~h}$. The slides of cells were collected for fixation and block, and then stained with primary antibody lba- $1(1: 100)$ at $4{ }^{\circ} \mathrm{C}$ for $16 \mathrm{~h}$. The next day, slides were incubated with fluorescent secondary antibody for $30 \mathrm{~min}$ at $37^{\circ} \mathrm{C}$ followed by a 5 min application of DAPI. Fluorescence images were captured with fluorescent microscopy (OLYMPUS-BX51).

\section{Cell culture}

BV-2 cells were purchased from the Wuxi Puhe Biomedical Technology Co., Ltd (Wuxi, China) and cultured in Dulbecco's Modified Eagle's Medium (DMEM) supplemented with $10 \%$ fetal bovine serum (FBS), $100 \mathrm{U} / \mathrm{mL}$ penicillin, and $100 \mu \mathrm{g} / \mathrm{mL}$ streptomycin under $5 \% \mathrm{CO}_{2}$ air environment at $37^{\circ} \mathrm{C}$.

\section{Oxygen-glucose-deprivation (OGD) model and treatments}

For the induction of OGD, BV-2 cells were incubated at $37^{\circ} \mathrm{C}$ in a hypoxic chamber (Sanyo Electric, Osaka, Japan) $\left(1 \% \mathrm{O}_{2}, 5 \% \mathrm{CO}_{2}\right)$ in glucose-free medium for different durations of time. Afterwards, glucose was replenished to normal levels and treated with EVs, EVs-miR-21a $a^{I N C}$ or EVs-miR-21a $a^{\text {inhibitor }}$ for $24 \mathrm{~h}$ under $5 \% \mathrm{CO}_{2}$ air environment at $37^{\circ} \mathrm{C}$ (reoxygenation).

\section{Cell Transfection}

MiR-21a-5p inhibitor and its inhibitor negative control (INC) were designed by GenePharma Corporation (Suzhou, China). MSCs were transfected miR-21a-5p inhibitor and INC by using a Lipofectamine 2,000 reagent (Invitrogen, Carlsbad, CA, USA) according to the manufacturer's instructions. At $6 \mathrm{~h}$ after transfection, the culture was continued with EVs-free serum medium for $24 \mathrm{~h}$ and then EVs-miR-21a INC and EVs-miR-21a $a^{\text {inhibitor }}$ were collected.

\section{RNase A and TritonX-100-treatment}

To degrade RNA, EVs were incubated in the presence of $20 \mu \mathrm{g} / \mathrm{mL}$ RNase A (Thermo Fisher Scientific, PA, USA) and/or with $0.5 \%$ TritonX-100 (Solarbio, Beijing, China) for $30 \mathrm{~min}$ at $37^{\circ} \mathrm{C}$. RNase digestion was stopped by addition $2 \mu \mathrm{L} / \mathrm{mL}$ RNase inhibitor (Thermo Scientific, PA, USA) and RNA-isolation was performed as previous described.

\section{Target prediction and Dual-Luciferase Reporter Assay}


The miR-21a-5p target genes were acquired using the following programs: TargetScanVert and miRDB. The prediction results show that STAT3 is one of the target genes of miR-21a-5p. The pmirGLO-STAT3 WT-3'-UTR or pmirGLO-STAT3 MUT-3'-UTR plasmids were purchased from Gene Pharma (Suzhou, China). Subsequently, the pmirGLO-WT/Mut-STAT3, miR-21a-5p mimics, and miR-21a-5p NC were all transfected into BV-2 cells according to the instructions of Lipo2000 (Thermo Fisher Scientific, PA, USA). Finally, DualLuciferase Reporter Assay System (Promage Corporation, USA) was used to detect firefly and renilla luciferase activities.

\section{Statistical analysis}

The SPSS software program was used to analyze the data. All values presented are expressed as the mean \pm standard deviation (SD). Data were analyzed with use of a one-way ANOVA and Bonferroni corrections for multiple post-hoc comparisons of means. A $p$ value $<0.05$ was required for results to be considered statistically significant. All "Ns" in each group for histological findings refer to the number of animals.

\section{Results}

\section{Characterization of MSCs and MSCs-EVs}

TEM analysis revealed that MSCs-EVs were almost around $100 \mathrm{~nm}$ and a rounded morphology (Figure 1A). MSCs-EVs were identified as small vesicles ranging from 60 to $120 \mathrm{~nm}$ with qNano (Figure 1B). MSCs-EVs expressed EVs markers such as CD9 and TSG10, with absence Calnexin, GM130, Cytochrome C1 or Lamin A/C with Western blot (Figure 1C). Additionally, PKH67-EVs were internalized in BV-2 cells and localized in the cytoplasm (Figure 1D).

MSCs exhibited a spindle-shaped morphology (Figure 1E) and were found to be capable of differentiating into osteoblasts and adipocytes (Figure 1F). MSCs were positive for CD44, CD29 and Sca-1, but negative for CD11b, CD45, CD31, CD117, Ly6G, CD19, CD14, or CD4 by FACS analysis (Figure 1G)

\section{MSCs-EVs treatment inhibited OGD-induced microglial apoptosis}

We first investigated whether MSCs-EVs exerted protective effects against OGD insult in BV-2 cells. The result showed that OGD followed by $24 \mathrm{~h}$ reoxygenation significantly decreased the viability of BV-2 cells at $1 \mathrm{~h}(\mathrm{t}=6.041, \mathrm{df}=10, p<0.01), 3 \mathrm{~h}(\mathrm{t}=8.095, \mathrm{df}=10, p<0.001)$ and $5 \mathrm{~h}(\mathrm{t}=10.364, \mathrm{df}=10, p<0.001)$ postOGD.

MSCs-EVs $(10,20 \mu \mathrm{g} / \mathrm{mL})$ increased the viability of the OGD-treated BV-2 cells partly at $3 \mathrm{~h}$ post-insult (Figure 2B) $(10 \mu \mathrm{g} / \mathrm{mL}: \mathrm{t}=-4.426, \mathrm{df}=10, p<0.01), 20 \mu \mathrm{g} / \mathrm{mL}:(\mathrm{t}=-9.253, \mathrm{df}=10, p<0.001)$. However, no significant differences exist at $5 \mu \mathrm{g} / \mathrm{mL}$ MSCs-EVs group $(\mathrm{t}=-1.591, \mathrm{df}=10, p>0.05)$ (Figure 2B). Further, in vitro experiments were performed using $10 \mu \mathrm{g} / \mathrm{mL}$ MSCs-EVs. 
When MSCs-EVs $(10 \mu \mathrm{g} / \mathrm{mL})$ were pretreated with RNase A did not affect the neuroprotection of MSCsEVs on cell viability at $3 \mathrm{~h}$ following OGD exposure $(p>0.05)$. In addition, pretreated MSCs-EVs with $0.5 \%$ Triton X-100 (detergent to lyse membrane vesicles) caused decrease in the protective properties of MSCsEVs $([F(4,25)=90.701, p<0.001]$; post hoc $p<0.001)$. And pretreated EVs with Triton X-100 and RNase A reversed the protective properties of MSCs-EVs on cell viability (post hoc $p<0.001$ ). Moreover, pretreated EVs with Triton X-100 and RNase A significantly decreased cell viability compared with Triton X-100 pretreatment (post hoc $p<0.001$ ) (Figure $2 \mathrm{C}$ ).

\section{MSCs-EVs treatment promoted M2 polarization in BV-2 cells following OGD exposure}

Because OGD induces microglia into a pro-inflammatory state, we examined the influence of EVs on BV-2 cells following OGD exposure. The qRT-PCR results showed that OGD exposure increased the levels of pro-inflammatory cytokines post-OGD, including TNFa $(\mathrm{t}=-4.034, \mathrm{df}=10, p<0.01$ at $1 \mathrm{~h} ; \mathrm{t}=-2.360, \mathrm{df}=10$, $p<0.05$ at $3 \mathrm{~h} ; \mathrm{t}=-1.902, \mathrm{df}=10, p>0.05$ at $5 \mathrm{~h}), \mathrm{IL}-1 \beta(\mathrm{t}=-3.124, \mathrm{df}=10, p<0.05$ at $1 \mathrm{~h} ; \mathrm{t}=-5.696, \mathrm{df}=10, p<0.01$ at $3 \mathrm{~h} ; \mathrm{t}=-4.932, \mathrm{df}=10, p<0.01$ at $5 \mathrm{~h}), \mathrm{NNOS}(\mathrm{t}=-0.393, \mathrm{df}=10, p>0.05$ at $1 \mathrm{~h} ; \mathrm{t}=-10.290, \mathrm{df}=10, p<0.001$ at 3 $h ; t=-4.490, \mathrm{df}=10, p<0.01$ at $5 \mathrm{~h})($ Figure $3 \mathrm{~A})$.

OGD dramatically decreased the levels of anti-inflammatory cytokines at $1 \mathrm{~h}, 3 \mathrm{~h}$, and $5 \mathrm{~h}$ post-insult: IL$10(\mathrm{t}=3.000, \mathrm{df}=10, p<0.05$ at $1 \mathrm{~h} ; \mathrm{t}=2.490, \mathrm{df}=10, p<0.05$ at $3 \mathrm{~h} ; \mathrm{t}=2.847, \mathrm{df}=10, p<0.05$ at $5 \mathrm{~h}), \mathrm{TGF}-\beta$ $(\mathrm{t}=5.071, \mathrm{df}=10, p<0.001$ at $1 \mathrm{~h} ; \mathrm{t}=3.936, \mathrm{df}=10, p<0.01$ at $3 \mathrm{~h} ; \mathrm{t}=5.737, \mathrm{df}=10, p<0.001$ at $5 \mathrm{~h}), \mathrm{M} 2$ microglia surface marker $\operatorname{CD} 206(\mathrm{t}=5.134, \mathrm{df}=10, p<0.001$ at $1 \mathrm{~h} ; \mathrm{t}=3.882, \mathrm{df}=10, p<0.01$ at $3 \mathrm{~h} ; \mathrm{t}=3.317$, $\mathrm{df}=10, p<0.01$ at $5 \mathrm{~h})$ (Figure $3 \mathrm{~A})$.

MSCs-EVs treatment attenuated the levels of pro-inflammatory cytokines at $3 \mathrm{~h}$ post-OGD, including TNFa $([\mathrm{F}(2,15)=157.208, p<0.001]$; post hoc $p<0.001), \mathrm{IL}-1 \beta([\mathrm{F}(2,15)=19.931, p<0.001]$; post hoc $p<0.01)$, and iNOS $([F(2,15)=104.828, p<0.001]$; post hoc $p<0.001)$ at $3 \mathrm{~h}$ following OGD insult (Figure 3B). Moreover, EVs treatment increased the levels of anti-inflammatory cytokines at $3 \mathrm{~h}$ post-OGD, including IL-10 $([F(2,15)=6.850, p<0.01]$; post hoc $p<0.05)$, TGF- $\beta([F(2,15)=7.367, p<0.01]$; post hoc $p<0.05)$ and $C D 206$ $([F(2,15)=17.170, p<0.001]$; post hoc $p<0.001)$ (Figure 3B). Taken together, these data indicate that MSCsEVs treatment are a homeostatic regulator of microglia polarization and facilitate the differentiation of microglia to $\mathrm{M} 2$, but not M1.

\section{miR-21a-5p in MSCs-EVs attenuated inflammatory response following OGD exposed in BV-2 cells}

EVs-mediated miRNA transport has been proposed to be an essential mechanism that regulates target gene expression for cell-to-cell communication [19]. We previously reported that miR-21a-5p was found to be highly abundant in MSCs-EVs [17]. Next, we observed that miR-21a-5p in MSCs-EVs account for its immune-modulatory property. First, in order to observe miR-21a-5p located in in the lumen, MSCs-EVs were treated with RNase A, Triton-X-100, and RNase A + TritonX-100 [20]. RT-qPCR analysis showed that pretreated MSCs-EVs with RNase A or Triton-X-100 alone did not abolish detection of miR-21a-5p in MSCs-EVs. Conversely, pretreated MSCs-EVs with RNase A and TritonX-100 could abolish the miR-21a-5p in MSCs-EVs (Figure 4A). These data confirmed that miR-21a-5p is inside of MSCs-EVs, which resistance 
to digestion. Following OGD exposure, miR-21a-5p levels were significantly decreased at $1 \mathrm{~h}(\mathrm{t}=2.687$, $\mathrm{df}=10, p<0.05), 3 \mathrm{~h}(\mathrm{t}=2.969, \mathrm{df}=10, p<0.05)$ and $5 \mathrm{~h}(\mathrm{t}=1.655, \mathrm{df}=10, p>0.05)$ post-HI (Figure 4B). MSCsEVs treatment remarkably augmented miR-21a-5p expression levels at $3 \mathrm{~h}$ post-OGD $([\mathrm{F}(2,15)=20.783$, p<0.001]; post hoc $p<0.001$ ) (Figures 4 C).

To confirm whether or not miR-21a-5p has a critical role in the aforementioned neuroprotection of MSCsEVs, MSCs-EVs were pretreated with an anti-miR-21a-5p inhibitor (EVs-miR-21a $a^{\text {inhibitor }}$ ) or with its negative control (EVs-miR-21a ${ }^{I N C}$ ). qRT-PCR results showed that miR-21a-5p inhibitor remarkable decreased miR21a-5p expression ( $[F(2,15)=162.026, p<0.001]$; post hoc $p<0.001)$ in MSCs-EVs (Figure 4D). Morever, miR-21a-5p inhibitor remarkable reversed EVs-induced miR-21a-5p levels post-OGD $([F(3,20)=15.773$, $p<0.001]$; post hoc $p<0.01$ ) (Figures $4 \mathrm{E}$ ).

EVs-miR-21a $a^{\text {inhibitor }}$ was significantly suppressed the cell viability following OGD in BV-2 cells as compare with EVs-miR-21a ${ }^{\mathrm{INC}},([\mathrm{F}(3,20)=31.199, p<0.001]$; post hoc $p<0.001)$ (Figure 4F).

EVs-miR-21a $a^{\text {inhibitor }}$ suppressed the anti-inflammatory effects of EVs-miR-21a ${ }^{N C}$ upon: TNF-a $([\mathrm{F}(3,20)=37.149, p<0.001]$; post hoc $p<0.001), \mathrm{IL}-1 \beta([\mathrm{F}(3,20)=23.528, p<0.001]$; post hoc $p<0.001)$, and iNOS $([F(3,20)=8.357, p<0.01]$; post hoc $p<0.05)$ and, while IL-10 $([F(3,20)=8.387, p<0.01]$; post hoc $p<0.05)$, TGF- $\beta([F(3,20)=14.381, p<0.001]$; post hoc $p<0.001)$ and $\operatorname{CD} 206([F(3,20)=12.117, p<0.001]$; post hoc $p<0.05$ ) expression was significantly increased following OGD exposure (Figure 4G).

\section{MiR-21a-5p in MSCs-EVs targets STAT3 signaling pathway following OGD exposed in BV-2 cells}

Next, to investigate the targets of miR-21a-5p that may be potentially associated with microglia polarization and inflammation, we used the available databases to search for potential miR-21a-5p targeted genes. Notably, the miR-21a-5p/STAT3 pathway has been shown to be the most critical cellular pathway related to the modulation of the immune system and inflammation $[21,22]$. Subsequently, we evidenced that OGD increased STAT3 phosphorylation ( $\mathrm{p}$-STAT3) at $1 \mathrm{~h}(\mathrm{t}=-4.209, \mathrm{df}=6 ; p<0.01), 3 \mathrm{~h}$ $(\mathrm{t}=-3.198, \mathrm{df}=6 ; p<0.05)$ and $5 \mathrm{~h}(\mathrm{t}=-4.348, \mathrm{df}=6 ; p<0.01)$ post-OGD (Figure $5 \mathrm{~A})$. MSCs-EVs treatment significantly decreased the levels p-STAT3 $([\mathrm{F}(3,12)=8.368, p<0.001]$; post hoc $p<0.05)$ (Figure $5 \mathrm{C})$. Furthermore, inhibition of miR-21a-5p in the MSCs-EVs group had exactly the reverse effect (post hoc $p<0.05$ ) (Figure 5C).

TargetScan analysis predicted binding of miR-21a-5p to 3'-UTR of STAT3 (Figure 5D). MiR-21a-5p mimics decreased luciferase activity associated with WT STAT3 3' UTR, but not luciferase activity associated with MUT STAT3 3' UTR in BV-2 cells $(\mathrm{t}=25.649, \mathrm{df}=10 ; p<0.001)$ (Figure 5E).

\section{MiR-21a-5p in MSCs-EVs promoted M2 microglia polarization following $\mathrm{HI}$ insult}

In order to confirm that the anti-inflammatory effect associated with MSCs-EVs administration is mediated by the EVs-delivery of miR-21a-5p, mice were injected with either PBS, MSCs-EVs, EVs-miR$21 a^{\text {inhibitor }}$ and EVs-miR-21 a ${ }^{I N C}$. Like that ours reported previously $[16,17]$, MSCs-EVs administration 
significantly decreased the water content $([\mathrm{F}(2,9)=50.806, p<0.001]$; post hoc $p<0.001)$ and infarct volumes $([\mathrm{F}(2,9)=189.879, p<0.001]$; post hoc $p<0.001)$ in $\mathrm{HI}$ neonatal mice (Figure $\mathrm{S} 1$ in supplementary material). EVs-miR-21a $a^{\text {inhibitor }}$ abrogated this effect of MSCs-EVs (Figure S2 in supplementary material).

Consistently, micrglia polarization was as assessed by immunofluorescence staining. The results revealed that the number of $\mathrm{M} 1$ phenotypes $\left(\mathrm{CD} 16^{+} / \mathrm{Iba} 1^{+}\right.$cells) the right cortex was significantly reduced (Figure $6 \mathrm{~A}$ ) and the number of $\mathrm{M} 2$ phenotypes (CD206 ${ }^{+} / \mathrm{Iba} 1^{+}$cells) in the ipsilateral cortex significantly increased (Figure 6B) in the $\mathrm{HI}+\mathrm{EV}$ s group as compared with that in response to $\mathrm{HI}$ alone when determined at $3 \mathrm{~d}$ post-HI.

\section{MiR-21a-5p in MSCs-EVs regulated STAT3 signaling pathway in the right cortex following $\mathrm{HI}$ insult}

Like that reported previously [22], p-STAT3 staining is localized in the $\mathrm{lba} 1^{+}$cells microglia/macrophages in the right hemisphere (Figure 7A). Consistent with in vitro data, EVs treatment significantly reduced $\mathrm{p}$ STAT3 $([\mathrm{F}(2,9)=17.488, p<0.01]$; post hoc $p<0.01)$ in the right cortex at $3 \mathrm{~d}$ post-HI (Figure 7B). EVs-miR$21 \mathrm{a}^{\text {inhibitor }}$ abrogated this effect of $\mathrm{EVs}([\mathrm{F}(3,12)=32.603, p<0.001]$; post hoc $p<0.01)$ (Figure $\left.7 \mathrm{C}\right)$.

\section{Discussion}

Recently, MSCs-EVs have been an attractive therapeutic approach for the treatment of inflammatory diseases and tissue injury. In this study, we found that MSCs-EVs alleviated neuroinflammation, associating with switch of microglia a pro- to an anti-inflammatory state following $\mathrm{HI}$ insult in vivo and in vitro. MiR-21a-5p shuttled by MSCs-EVs might participate in microglia polarization by targeting STAT3 to regulate neuroinflammation and maintain brain homeostasis.

\section{MSCs-EVs suppressed neuroinflammation by switch of microglia from a pro- to an anti-inflammatory state}

Therapeutic effects of MSCs-EVs in ischemic brain are related to its immunosuppressive properties. MSCs-derived microvesicles dampened LPS-induced inflammatory responses in BV-2 cells [23]. MSCsEVs suppressed the microglia activation, switched microglia toward an anti-inflammatory phenotype, increased dendritic spine density in experiment Alzheimer's disease animal [24]. MSCs-derived EVs suppressed early inflammatory responses by modulating microglia/macrophage polarization after traumatic brain injury in rat [25]. Umbilical cord MSCs-derived EVs are internalized by microglia cells and dampened neuroinflammation following $\mathrm{HI}$ brain damage in newborn rats [15]. MSCs-EVs treatment significantly reduced microgliosis and prevented reactive astrogliosis following LPS-stimulated brain injury [26]. We previous found that MSCs-EVs by intracardial injection were found to be localized in the microglia after $\mathrm{HI}$ insult in vivo and in vitro $[16,17]$. MSCs-EVs treatment reduced neuroinflammation by suppressing osteopontin expression in microglia/macrophage after $\mathrm{HI}$ insult in neonatal mice[27]. In the current study, MSCs-EVs, internalized in microglia, decreased BV-2 cells apoptosis, reduced the expression of the M1 microglial markers (including IL-1 $\beta$, iNOS and TNF-a), while increased the expression of the M2 
microglial markers (including CD206, TGF $\beta$ and CD206) following OGD BV-2 cells. Confirmed with in vitro study, we found that MSCs-EVs treatment reduced the number of M1 phenotypes (CD $16^{+} / \mathrm{lba} 1^{+}$cells), while increased the number of M2 phenotypes (CD206 $/ / \mathrm{lba} 1^{+}$cells) in the ipsilateral cortex, indicating that MSCs-EVs might be a promising therapeutic treatment for $\mathrm{HI}$ brain damage by modulating microglial polarization.

\section{Anti-inflammatory mechanisms of miR-21a-5p in MSCs-EVs}

One of the main mechanisms responsible for the therapeutic properties of EVs injected in the brain ischemia is the transfer of miRNAs between cells. We previously reported that miR-21a-5p was found to be highly abundant in MSCs-EVs [17]. Several studies indicated that miR-21 play important role in the anti-inflammatory response in inflammatory-related diseases, tumor, infection, and so on [28, 29] . MiR-21 overexpression markedly inhibited the inflammatory cytokine production and improved cardiac dysfunction after myocardial infarction [30]. EVs from hypoxia-preconditioned MSCs rescued synaptic dysfunction and regulated inflammatory responses to improve learning and memory function via replenishment of miR-21 in Alzheimer disease animal modle [31]. Moreover, overexpression of miR-21 in EVs remarkedly reduced cell apoptosis and improved cardiac function after myocardial infarction [32]. We found that MSCs-EVs treatment markedly increased OGD-reduced miR-21a-5p expression in BV-2 cells. MiR-21a-5p inhibitor abrogated the beneficial effects of MSCs-EVs on microglial polarization, inflammatory cytokines and cell survival, showing that miR-21a-5p transmitted by MSCs-EVs play considerably role in neuroprotective effect on $\mathrm{HI}$ exposure.

\section{STAT3 signaling pathway contributed to MSCs-EVs' effect on microglial M2 polarization}

The STAT3 signaling pathway is important for cellular growth, differentiation, and survival [33], involved in a variety of inflammatory and anti-inflammatory responses[34]. Activated STAT3 was predominantly localized in the macrophages/microglia in the post-ischemic brain [22]. Activation of microglial STAT3 induced expression of pro-inflammatory factors following cerebral ischemia $[35,36]$. In the present study, STAT3 was activated after $\mathrm{HI}$ insult in vivo and in vitro, in consistent with increased pro-inflammatory cytokines, decreased anti-inflammatory cytokines. MSCs-EVs treatment suppressed STAT3 activation, in consistent with switch of microglia a pro- to an anti-inflammatory state following $\mathrm{HI}$ insult in vivo and in vitro. After confirming a direct binding by Dual-Luciferase Reporter Assay, we found a negative correlation between STAT3 and miR-21a-5p after OGD in microglia. In addition, miR-21a-5p inhibitor abrogated STAT3 activation in microglia in vivo and in vitro. Taken together, the data suggested that MSCs-EVs regulated microglial activation by transferring miR-21a-5p to microglia and targeting STAT3 pathway.

This study has several limitations. First, the level of miR-21a-5p was decreased after OGD exposure in microglia. We previously reported that the level of miR-21a-5p decreased in the right hemisphere at $24 \mathrm{~h}$ and $48 \mathrm{~h}$ post-HI, whereas increased at $72 \mathrm{~h}$ post-HI insult. We did not observe the miR-21a-5p expression in microglia in vivo. Additionally, limited intracardial injection of MSCs-EVs administrations $(24 \mathrm{~h}$ after $\mathrm{HI}$ 
insult) was tested in this study. Whether multiple treatments of MSCs-EVs using different time courses or modes of administration would produce better effects against $\mathrm{HI}$ will require future investigations.

\section{Conclusion}

In summary, the findings showed that MSCs-EVs treatment attenuated $\mathrm{HI}$ brain damage in neonatal mice via shuttling miR-21a-5p, which induced microglia M2 polarization by targeting STAT3.

\section{List Of Abbreviations}

CNS, central nervous system

DAPI, 4',6'-diamidino-2-phenylindole dihydrochloride hydrate

EVs, extracellular vesicles

$\mathrm{HI}$, hypoxic-ischemic

Iba-1, ionized calcium binding adapter molecule 1

MSCs, mesenchymal stem cells

OGD, oxygen-glucose deprivation

qRT-PCR, Reverse transcriptase quantitative real-time PCR

STAT3, signal transducer and activator of transcription 3

TTC, 2,3,5-triphenyltetrazolium chloride monohydrate

\section{Declarations}

\section{Competing Interest}

The authors have no financial or personal conflict of interest to disclose.

\section{Ethics Approval and Consent to participate}

All animal experiments were conducted in accordance with the International Guiding Principles for Animal Research provided by the Council for International Organizations of Medical Sciences (CIOMS), and the procedures were approved by the Animal Ethical and Welfare Committee of Shandong University (approval No. ECSBMSSDU2018-2-059). Laboratory operators were trained in accordance with Institutional Animal Care and Use Committee Guidebook (IACUC) rules. 
Not applicable. This article does not contain any individual person's data.

\section{Availability of data and materials}

The datasets used and analyzed during the current study are available from the corresponding author on reasonable request.

\section{Competing Interest}

The authors have no financial or personal conflict of interest to disclose.

\section{Funding}

Research funding support for this work was from the National Natural Science Foundation of China (No. 81873768,82072535 and 81671213 to Dr. Zhen Wang), The National Key Research and Development Program of China (No. 2017 YFC0820203 to Dr. Dexiang Liu). The national key project of chronic noncommunicable disease of China (No.2016YFC1300403 to Dr. Wenqiang Chen) and National Natural Science Foundation of China (No.81770436 to Dr. Wenqiang Chen).

\section{Authors' contribution}

ZW: made substantial contributions to study design, data interpretation, writing and revising of the manuscript, and final revision of the manuscript; DXL: conceived experiments, analyzed the data and final revision of the manuscript; DQX: made substantial contributions to laboratory work, analyzed the data, and edited the manuscript; YJZ: performed Western blot; TTL, HFK, and CCG performed cell culture, XFG and WQC: revised the manuscript. The authors have no conflict of interest to declare. All authors read and approved the final manuscript.

\section{Acknowledgements}

We thanks the animal medicine center of Shandong University for providing experimental animals, and the Basic Medical Sciences of Shandong University for providing experimental places and instrument.

\section{References}

1. Ziemka-Nalecz, M., J. Jaworska, and T. Zalewska, Insights Into the Neuroinflammatory Responses After Neonatal Hypoxia-Ischemia. J Neuropathol Exp Neurol, 2017. 76(8): p. 644-654.

2. Hagberg, H., et al., The role of inflammation in perinatal brain injury. Nat Rev Neurol, 2015. 11(4): p. 192-208.

3. Liu, F. and L.D. McCullough, Inflammatory responses in hypoxic ischemic encephalopathy. Acta Pharmacol Sin, 2013. 34(9): p. 1121-30.

4. Colton, C.A., Heterogeneity of microglial activation in the innate immune response in the brain. $\mathrm{J}$ Neuroimmune Pharmacol, 2009. 4(4): p. 399-418. 
5. Bernardo, M.E. and W.E. Fibbe, Mesenchymal stromal cells: sensors and switchers of inflammation. Cell Stem Cell, 2013. 13(4): p. 392-402.

6. Yin, Y., et al., Human umbilical cord-derived mesenchymal stem cells direct macrophage polarization to alleviate pancreatic islets dysfunction in type 2 diabetic mice. Cell Death Dis, 2018. 9(7): p. 760.

7. Bazzoni, R., et al., Extracellular Vesicle-Dependent Communication Between Mesenchymal Stromal Cells and Immune Effector Cells. Frontiers in Cell and Developmental Biology, 2020. 8.

8. Crisostomo, P.R., et al., Human mesenchymal stem cells stimulated by TNF-alpha, LPS, or hypoxia produce growth factors by an NF kappa B- but not JNK-dependent mechanism. Am J Physiol Cell Physiol, 2008. 294(3): p. C675-82.

9. Bazzoni, R., et al., Extracellular Vesicle-Dependent Communication Between Mesenchymal Stromal Cells and Immune Effector Cells. Front Cell Dev Biol, 2020. 8: p. 596079.

10. Thery, C., et al., Minimal information for studies of extracellular vesicles 2018 (MISEV2018): a position statement of the International Society for Extracellular Vesicles and update of the MISEV2014 guidelines. J Extracell Vesicles, 2018. 7(1): p. 1535750.

11. Deng, M., et al., Mesenchymal Stem Cell-Derived Extracellular Vesicles Ameliorates Hippocampal Synaptic Impairment after Transient Global Ischemia. Front Cell Neurosci, 2017. 11: p. 205.

12. Ophelders, D.R., et al., Mesenchymal Stromal Cell-Derived Extracellular Vesicles Protect the Fetal Brain After Hypoxia-Ischemia. Stem Cells Transl Med, 2016. 5(6): p. 754-63.

13. Thomi, G., et al., Intranasally Administered Exosomes from Umbilical Cord Stem Cells Have Preventive Neuroprotective Effects and Contribute to Functional Recovery after Perinatal Brain Injury. Cells, 2019. 8(8).

14. Joerger-Messerli, M.S., et al., Extracellular Vesicles Derived from Wharton's Jelly Mesenchymal Stem Cells Prevent and Resolve Programmed Cell Death Mediated by Perinatal Hypoxia-Ischemia in Neuronal Cells. Cell Transplant, 2018. 27(1): p. 168-180.

15. Thomi, G., et al., Exosomes derived from umbilical cord mesenchymal stem cells reduce microgliamediated neuroinflammation in perinatal brain injury. Stem Cell Res Ther, 2019. 10(1): p. 105.

16. Chu, X., et al., Hydrogen sulfide-modified extracellular vesicles from mesenchymal stem cells for treatment of hypoxic-ischemic brain injury. J Control Release, 2020. 328: p. 13-27.

17. Xin, D., et al., Mesenchymal stromal cell-derived extracellular vesicles modulate microglia/macrophage polarization and protect the brain against hypoxia-ischemic injury in neonatal mice by targeting delivery of miR-21a-5p. Acta Biomater, 2020. 113: p. 597-613.

18. Wang, F.W., et al., Roles of activated astrocytes in bone marrow stromal cell proliferation and differentiation. Neuroscience, 2009. 160(2): p. 319-29.

19. Chevillet, J.R., et al., Quantitative and stoichiometric analysis of the microRNA content of exosomes. Proc Natl Acad Sci U S A, 2014. 111(41): p. 14888-93.

20. Ridder, K., et al., Extracellular vesicle-mediated transfer of functional RNA in the tumor microenvironment. Oncoimmunology, 2015. 4(6): p. e1008371. 
21. Fang, Y.Y. and J.H. Zhang, MFG-E8 alleviates oxygen-glucose deprivation-induced neuronal cell apoptosis by STAT3 regulating the selective polarization of microglia. Int J Neurosci, 2020: p. 1-10.

22. Satriotomo, I., K.K. Bowen, and R. Vemuganti, JAK2 and STAT3 activation contributes to neuronal damage following transient focal cerebral ischemia. J Neurochem, 2006. 98(5): p. 1353-68.

23. Jaimes, Y., et al., Mesenchymal Stem Cell-Derived Microvesicles Modulate LipopolysaccharidesInduced Inflammatory Responses to Microglia Cells. Stem Cells, 2017. 35(3): p. 812-823.

24. Losurdo, M., et al., Intranasal delivery of mesenchymal stem cell-derived extracellular vesicles exerts immunomodulatory and neuroprotective effects in a 3xTg model of Alzheimer's disease. Stem Cells Transl Med, 2020. 9(9): p. 1068-1084.

25. Ni, H., et al., Exosomes Derived From Bone Mesenchymal Stem Cells Ameliorate Early Inflammatory Responses Following Traumatic Brain Injury. Front Neurosci, 2019. 13: p. 14.

26. Drommelschmidt, $\mathrm{K}$., et al., Mesenchymal stem cell-derived extracellular vesicles ameliorate inflammation-induced preterm brain injury. Brain Behav Immun, 2017. 60: p. 220-232.

27. Xin, D., et al., MSCs-extracellular vesicles attenuated neuroinflammation, synapse damage and microglial phagocytosis after hypoxia-ischemia injury by preventing osteopontin expression. Pharmacol Res, 2020: p. 105322.

28. Sheedy, F.J., et al., Negative regulation of TLR4 via targeting of the proinflammatory tumor suppressor PDCD4 by the microRNA miR-21. Nat Immunol, 2010. 11(2): p. 141-7.

29. Sheedy, F.J., Turning 21: induction of miR-21 as a key swith in the inflammatory response. Frontiers in Immunology, 2015. 6.

30. Yang, L.S., et al., MicroRNA-21 prevents excessive inflammation and cardiac dysfunction after myocardial infarction through targeting KBTBD7. Cell Death \& Disease, 2018. 9.

31. Cui, G.H., et al., Exosomes derived from hypoxia-preconditioned mesenchymal stromal cells ameliorate cognitive decline by rescuing synaptic dysfunction and regulating inflammatory responses in APP/PS1 mice. FASEB J, 2018. 32(2): p. 654-668.

32. Song, Y., et al., Localized injection of miRNA-21-enriched extracellular vesicles effectively restores cardiac function after myocardial infarction. Theranostics, 2019. 9(8): p. 2346-2360.

33. Yu, H., D. Pardoll, and R. Jove, STATs in cancer inflammation and immunity: a leading role for STAT3. Nature Reviews Cancer, 2009. 9(11): p. 798-809.

34. Rawlings, J.S., K.M. Rosler, and D.A. Harrison, The JAK/STAT signaling pathway. Journal of Cell Science, 2004. 117(8): p. 1281-1283.

35. Satriotomo, I., K.K. Bowen, and R. Vemuganti, JAK2 and STAT3 activation contributes to neuronal damage following transient focal cerebral ischemia. Journal of Neurochemistry, 2006. 98(5): p. 1353-1368.

36. Li, R., et al., TSG-6 attenuates inflammation-induced brain injury via modulation of microglia polarization in SAH rats through the SOCS3/STAT3 pathway. Journal of Neuroinflammation, 2018. 15. 
A

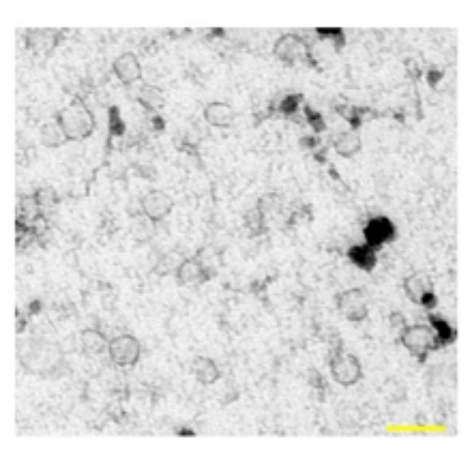

B

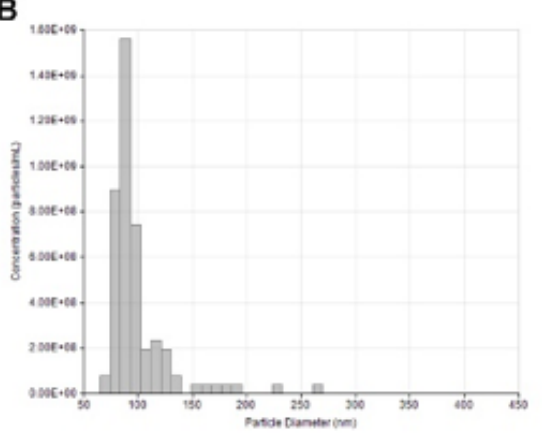

c
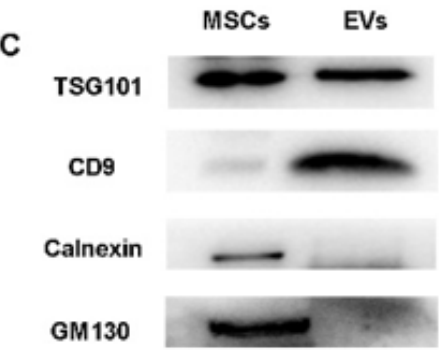

Cytochrome $\mathrm{C1}$

Lamin A/C
D

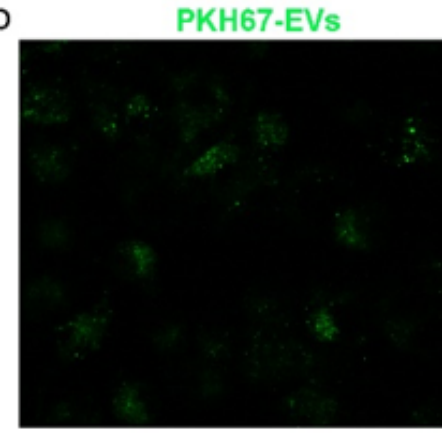

E

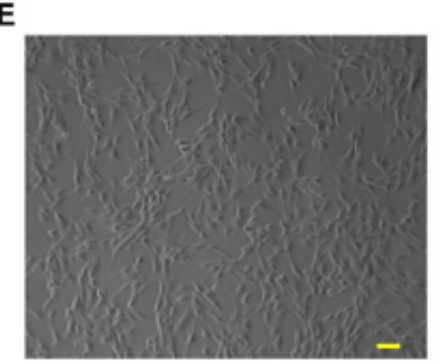

G
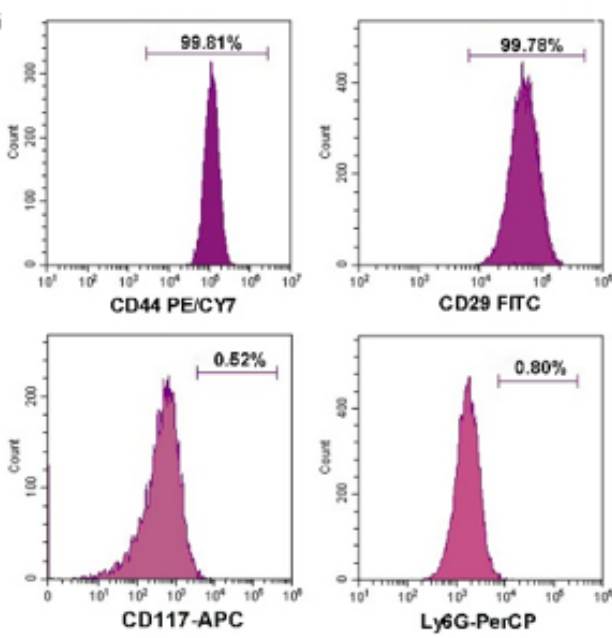

F
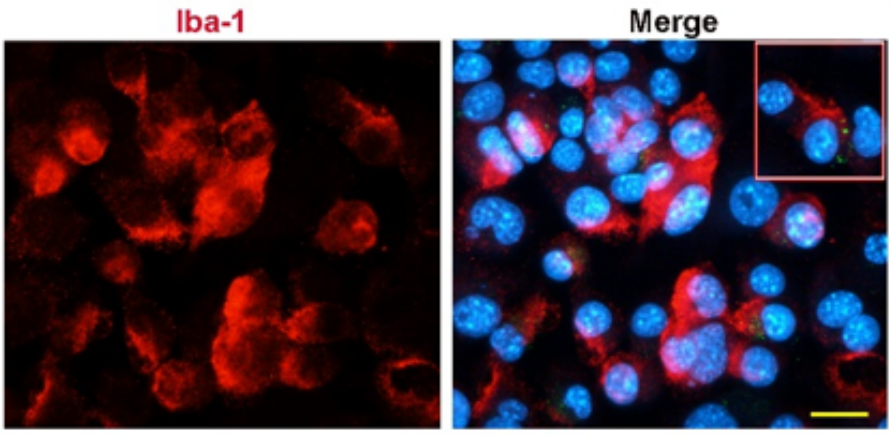

D1
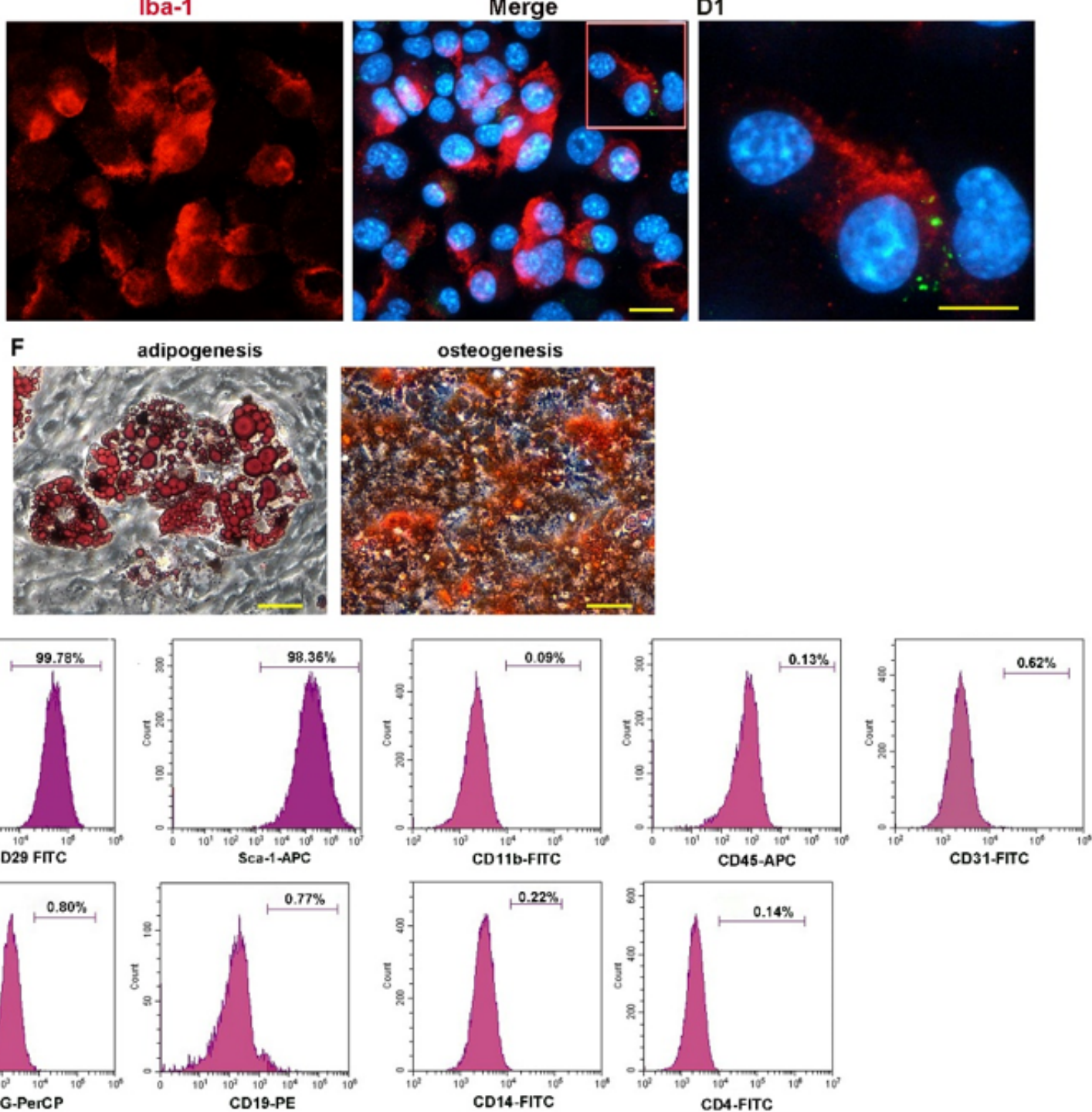

\section{Figure 1}

Identification of EVs and MSCs (A) Electron microscopic image of EVs. Scale bar = $200 \mathrm{~nm}$. (B) Results of nanoparticle tracking analysis of MSCs-EVs. (C) Western blot to observe the expression of TSG101, CD9, GM130, Lamin A/C, Cytochirome C1 and Calnexin in MSCs and EVs. (D) Immunofluorescent analyses of 
EVs invasion into BV-2 cells as determined at $3 \mathrm{~h}$ post-OGD. Scale bar $=20 \mu \mathrm{m}$. (D1) Magnified views of boxed regions in D showing the location of PKH67 hotspots. Scale Bar $=5 \mu \mathrm{m}$. (E) MSCs showed a typical spindle-like morphology. Scale bar $=20 \mu \mathrm{m}$. (F) MSCs showed potential differentiation capacity for adipogenesis and osteogenesis. Scale bar $=100 \mu \mathrm{m}$. (G) FACS of characteristic MSCs surface markers.

A

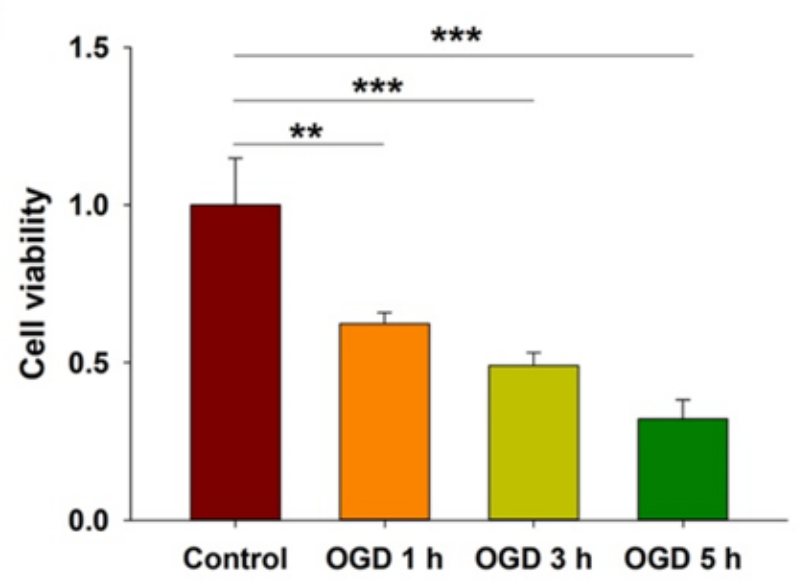

C

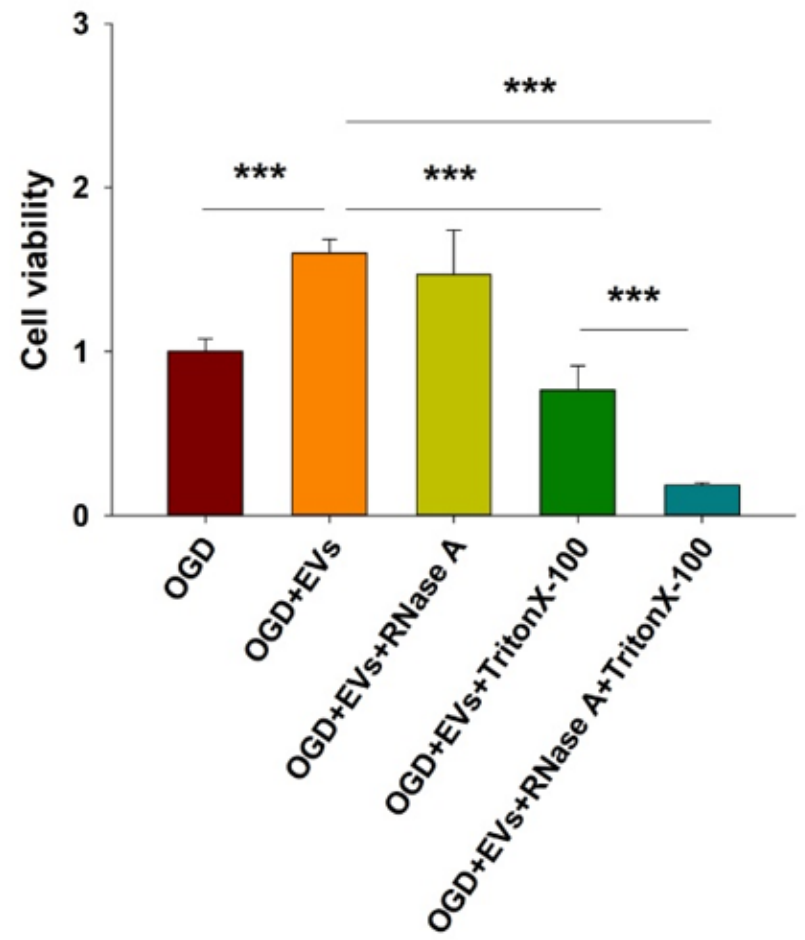

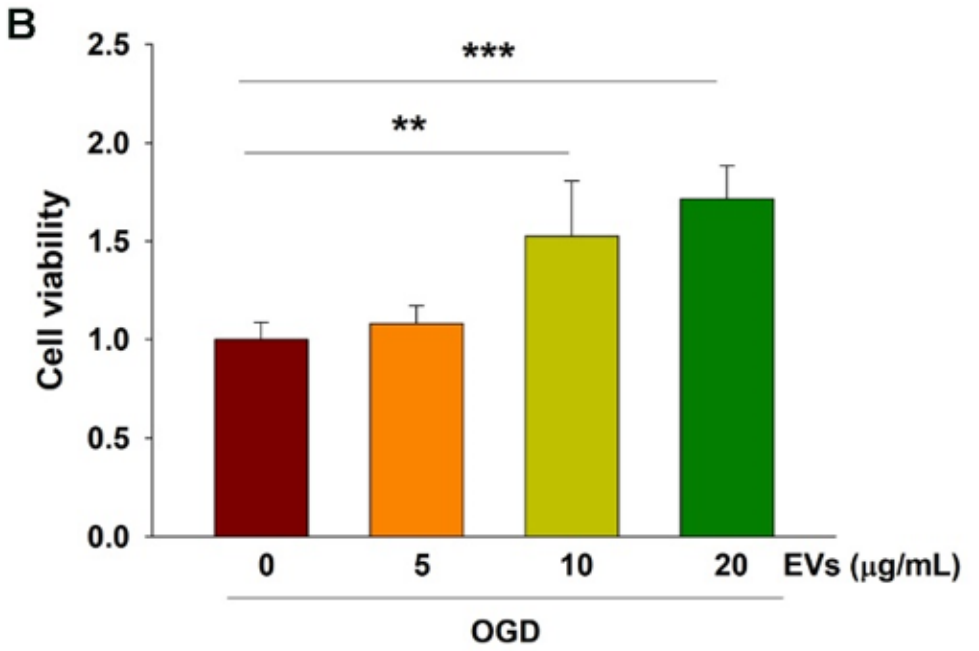

\section{Figure 2}

Effects of MSCs-EVs on OGD-induced microglial apoptosis (A) The viability of BV-2 cells was measured at $1 \mathrm{~h}, 3 \mathrm{~h}$ and $5 \mathrm{~h}$ OGD following $24 \mathrm{~h}$ reperfusion. $\mathrm{N}=6$ /group. (B) At $3 \mathrm{~h}$ OGD following $24 \mathrm{~h}$ reoxygenation, the viability of BV-2 cells was measured in the presence or absence of MSCs-EVs. $\mathrm{N}=6 /$ group. (C) At $3 \mathrm{~h}$ OGD following $24 \mathrm{~h}$ reoxygenation, the cell viability was measured in BV-2 cells that were treated with naive MSCs-EVs, MSCs-EVs pretreated with RNase A (+RNase A) or TritonX-100 
(+TritonX-100) or both of them (+RNase A+TritonX-100) N=6/group. Cells incubated under standard cell culture conditions ("Control”) were defined as $100 \%$ cell survival. Values represent the mean \pm SD, ${ }^{* *} \mathrm{p}<0.01,{ }^{* *} \mathrm{p}<0.001$ according to t-test in $\mathrm{A}$ and $\mathrm{B} .{ }^{* * *} \mathrm{p}<0.001$ according to ANOVA with Bonferroni correction in $\mathrm{C}$.

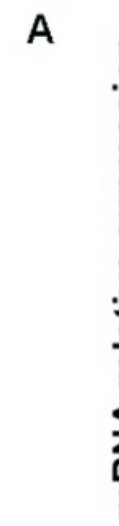

B
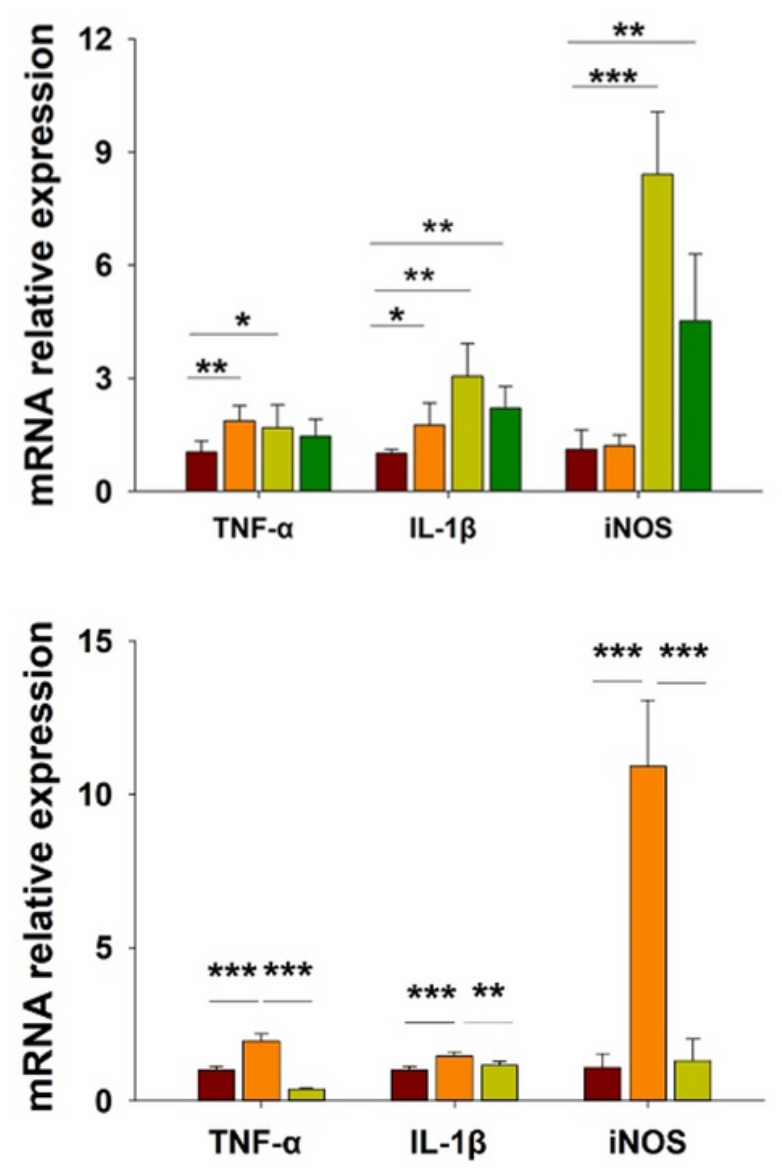

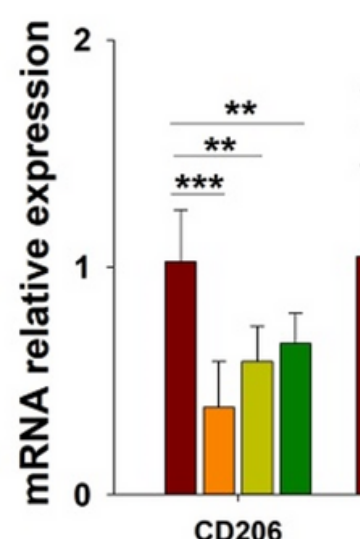

CD206
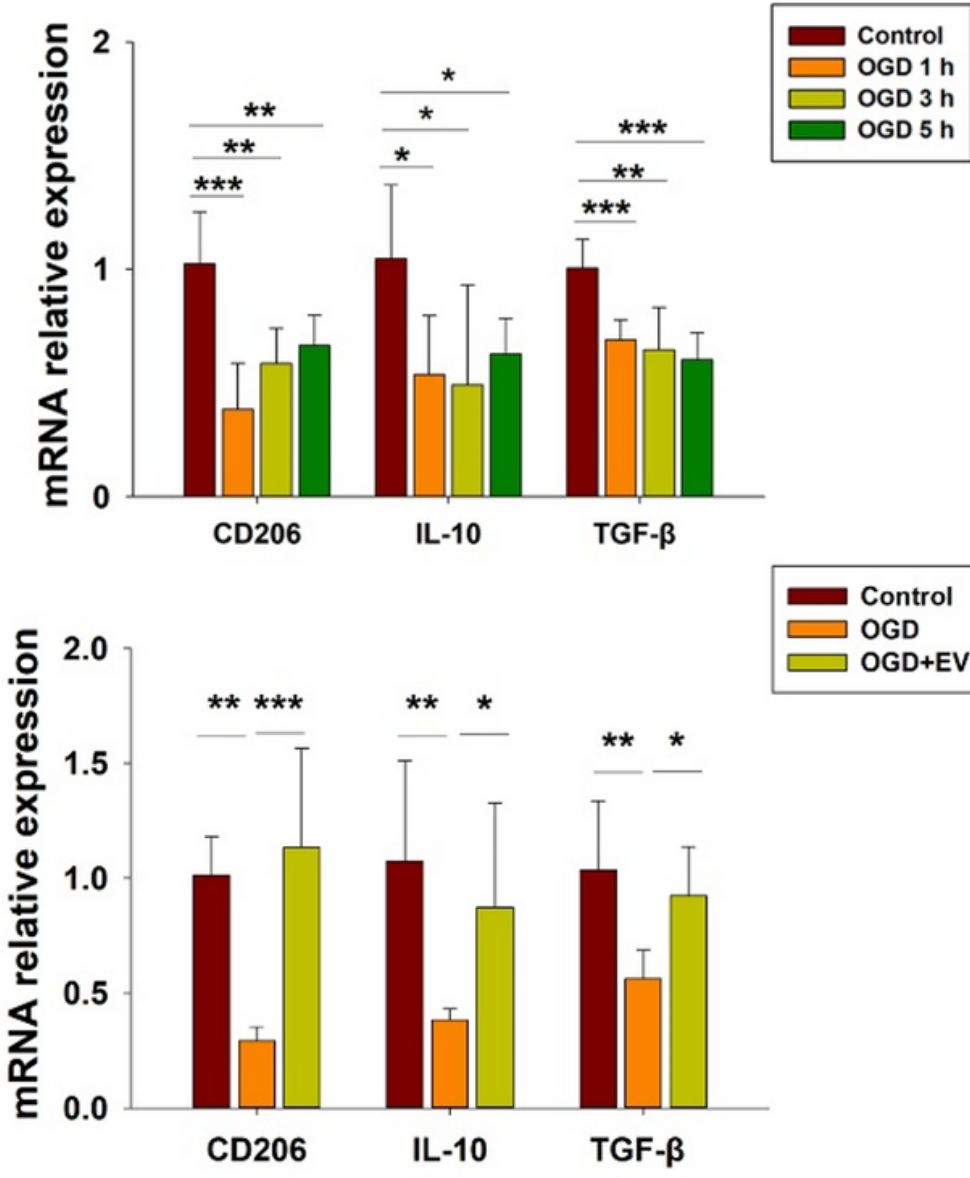

0.0

\section{Figure 3}

EVs promoted M2 polarization in BV-2 cells following OGD exposure (A) The pro- and anti-inflammatory mRNA expressions in BV-2 cells at $1 \mathrm{~h}, 3 \mathrm{~h}$ and $5 \mathrm{~h}$ OGD following $24 \mathrm{~h}$ reoxygenation with use of qRTPCR. $N=6$ /group. (B) The mRNA levels of IL-1 $\beta$, iNOS, TNFa, IL-10, TGF- $\beta$, and CD206 were measured in the presence or absence of MSCs-EVs at $3 \mathrm{~h}$ OGD following $24 \mathrm{~h}$ reoxygenation. $\mathrm{N}=6 /$ group. Values represent the mean $\pm S D,{ }^{*} p<0.05,{ }^{\star \star} p<0.01,{ }^{* \star *} p<0.001$ according to t-test in $A .{ }^{*} p<0.05,{ }^{\star \star} p<0.01$, $\star \star \star x<0.001$ according to ANOVA with Bonferroni correction in B. 

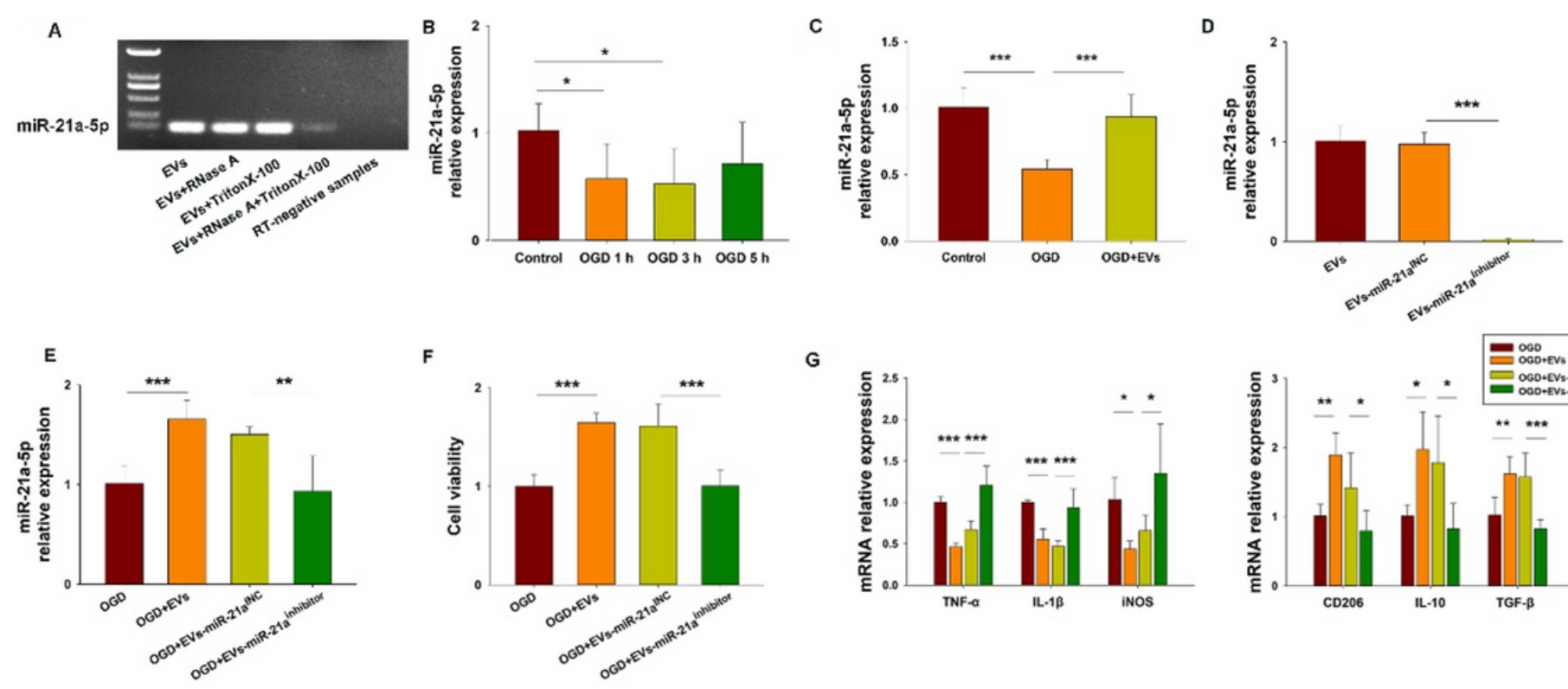

G
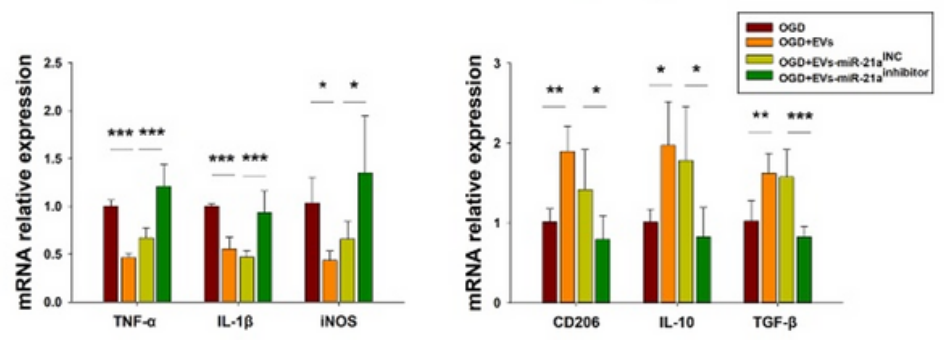

\section{Figure 4}

MiR-21a-5p in MSCs-EVs attenuated inflammatory response following OGD exposed in BV-2 cells (A) Levels of miR-21a-5p were detected in MSCs-EVs that were pretreated with RNase A, TritonX-100, and TritonX-100 + RNase A. (B) The levels of miR-21a-5p was measured at $1 \mathrm{~h}, 3 \mathrm{~h}$ and $5 \mathrm{~h}$ OGD following 24 $\mathrm{h}$ reoxygenation with use of qRT-PCR. $\mathrm{N}=6 /$ group. (C) The levels of miR-21a-5p were measured in the presence or absence of MSCs-EVs at $3 \mathrm{~h}$ OGD following $24 \mathrm{~h}$ reoxygenation. $\mathrm{N}=6 / \mathrm{group}$. (D) The levels of miR-21a-5p in the corresponding EVs (EVs, EVs-miR-21aINC and EVs-miR-21ainhibitor) were detected with use of qRT-PCR. $N=6 /$ group. (E) The levels of miR-21a-5p were measured in each group as treated with EVs, EVs-miR-21ainhibitor, or EVs-miR-21aINC at $3 \mathrm{~h}$ OGD following $24 \mathrm{~h}$ reoxygenation. $\mathrm{N}=6 /$ group. (F) At $3 \mathrm{~h}$ OGD following $24 \mathrm{~h}$ reoxygenation, the cell viability of BV-2 cells was measured in each group as treated with EVs, EVs-miR-21ainhibitor, or EVs-miR-21aINC. $N=6 /$ group. (G) The mRNA levels of IL-1 $\beta$, iNOS, TNFa, IL-10, TGF- $\beta$, YM1 and CD206 were measured in the presence or absence of EVs, EVs-miR21ainhibitor, EVs-miR-21aINC at $3 \mathrm{~h}$ OGD following $24 \mathrm{~h}$ reperfusion. $\mathrm{N}=6$ /group. Values represent the mean $\pm S D,{ }^{*} p<0.05$ according to t-test in $B .{ }^{*} p<0.05,{ }^{*} p<0.01,{ }^{* \star *} p<0.001$ according to ANOVA with Bonferroni correction in $\mathrm{C}$ to $\mathrm{G}$. 
A

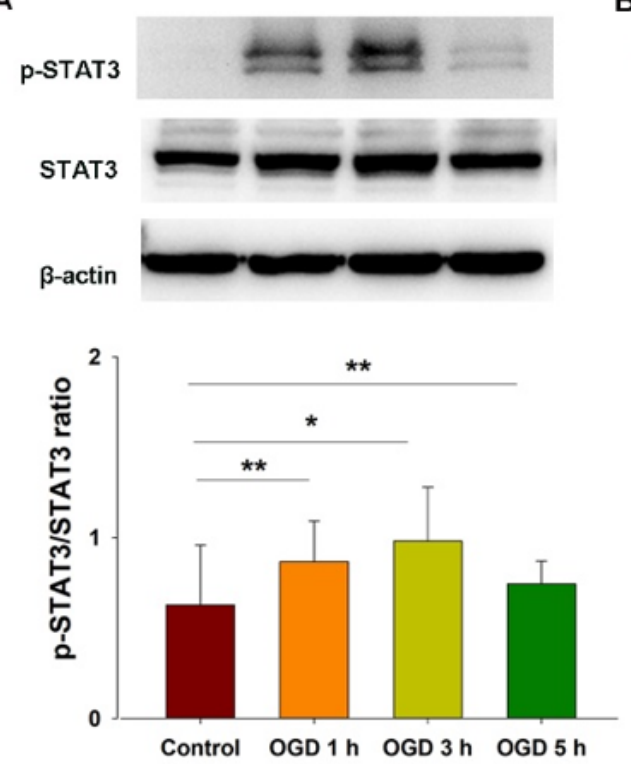

B
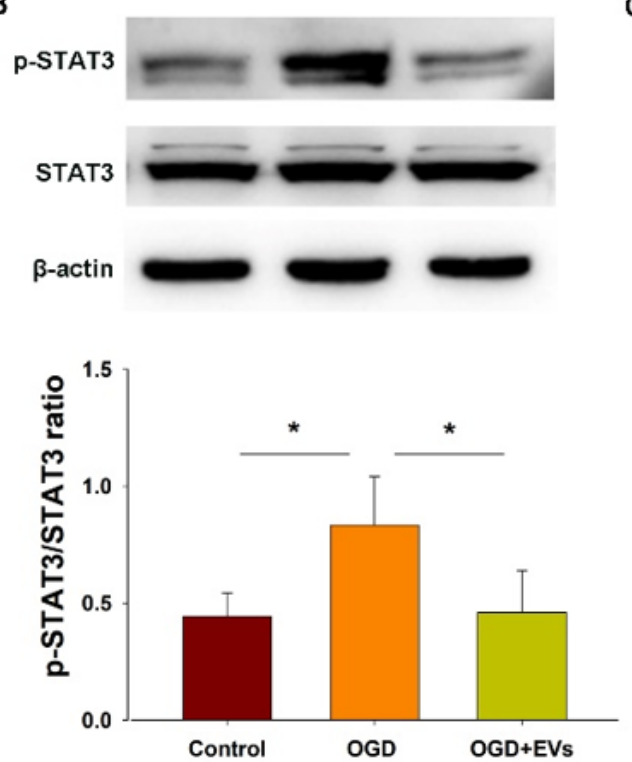

$E$

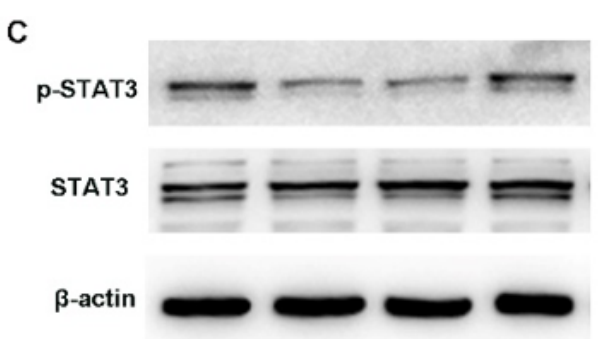

D
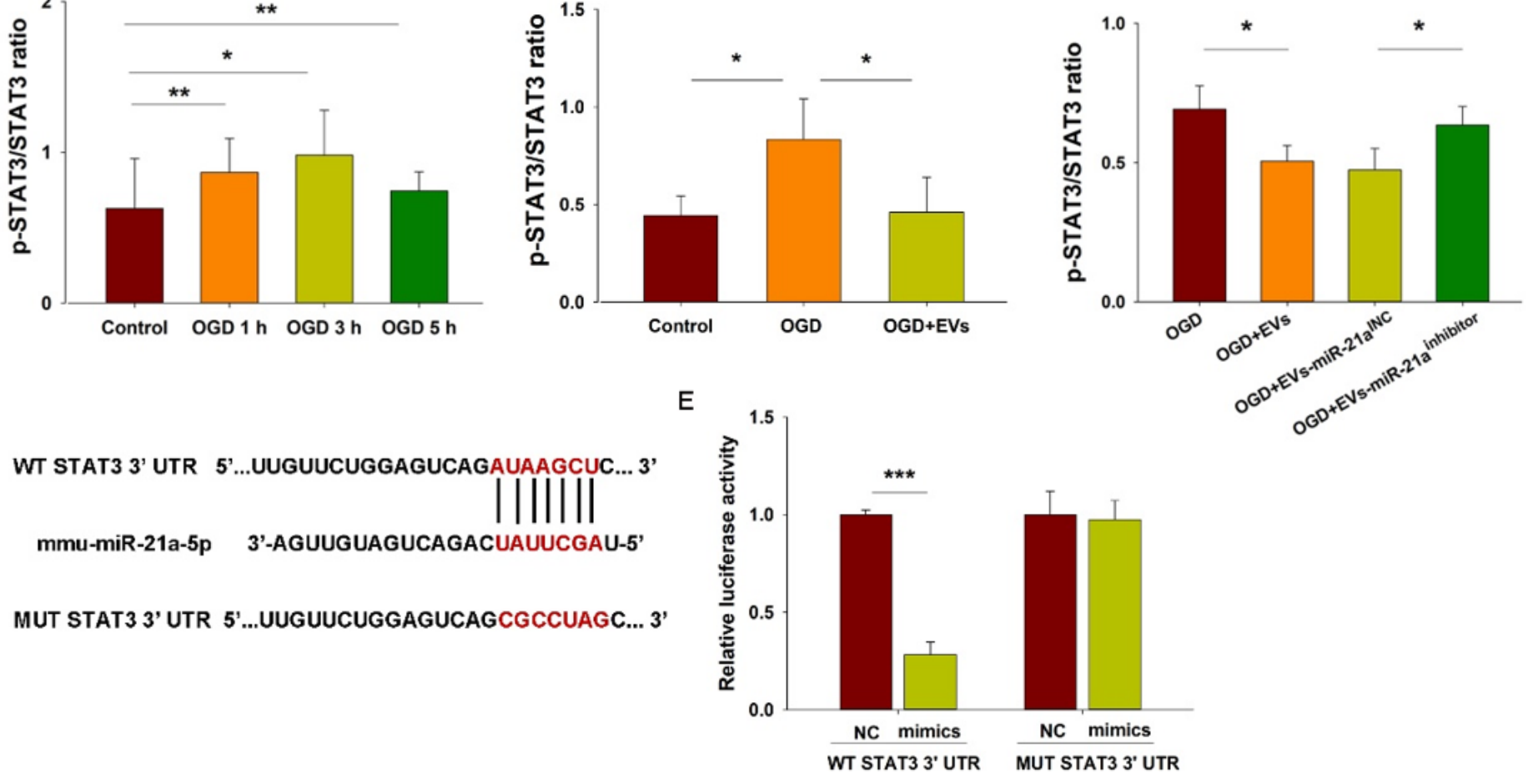

\section{Figure 5}

MiR-21a-5p in MSCs-EVs targets STAT3 signaling pathway following OGD exposed in BV-2 cells (A) The levels of STAT3 phosphorylation (p-STAT3) and STAT3 were measured at $1 \mathrm{~h}, 3 \mathrm{~h}$ and $5 \mathrm{~h}$ OGD following $24 \mathrm{~h}$ reoxygenation with Western bolt. $\mathrm{N}=4$ /group. (B) The levels of STAT3 phosphorylation (p-STAT3) and STAT3 were measured in the presence or absence of MSCs-EVs at $3 \mathrm{~h}$ OGD following $24 \mathrm{~h}$ reoxygenation. $\mathrm{N}=4$ /group. (C) Target regions of miR-21a-5p and STAT3. (D) Effects up- or downregulation of miR-21a-5p on luciferase activities of wild type (WT) or mutated (MUT) STAT3 reporter as determined by Dual-Luciferase Reporter Assay in BV-2 cells at $48 \mathrm{~h}$ after transfection. $\mathrm{N}=6 /$ group. Values represent the mean $\pm S D,{ }^{*} p<0.05,{ }^{* *} p<0.01,{ }^{* \star *} p<0.001$ according to ANOVA with $t$-test in $A$ and $E$. ${ }^{*} \mathrm{p}<0.05$ according to ANOVA with Bonferroni correction in B and $\mathrm{C}$. 


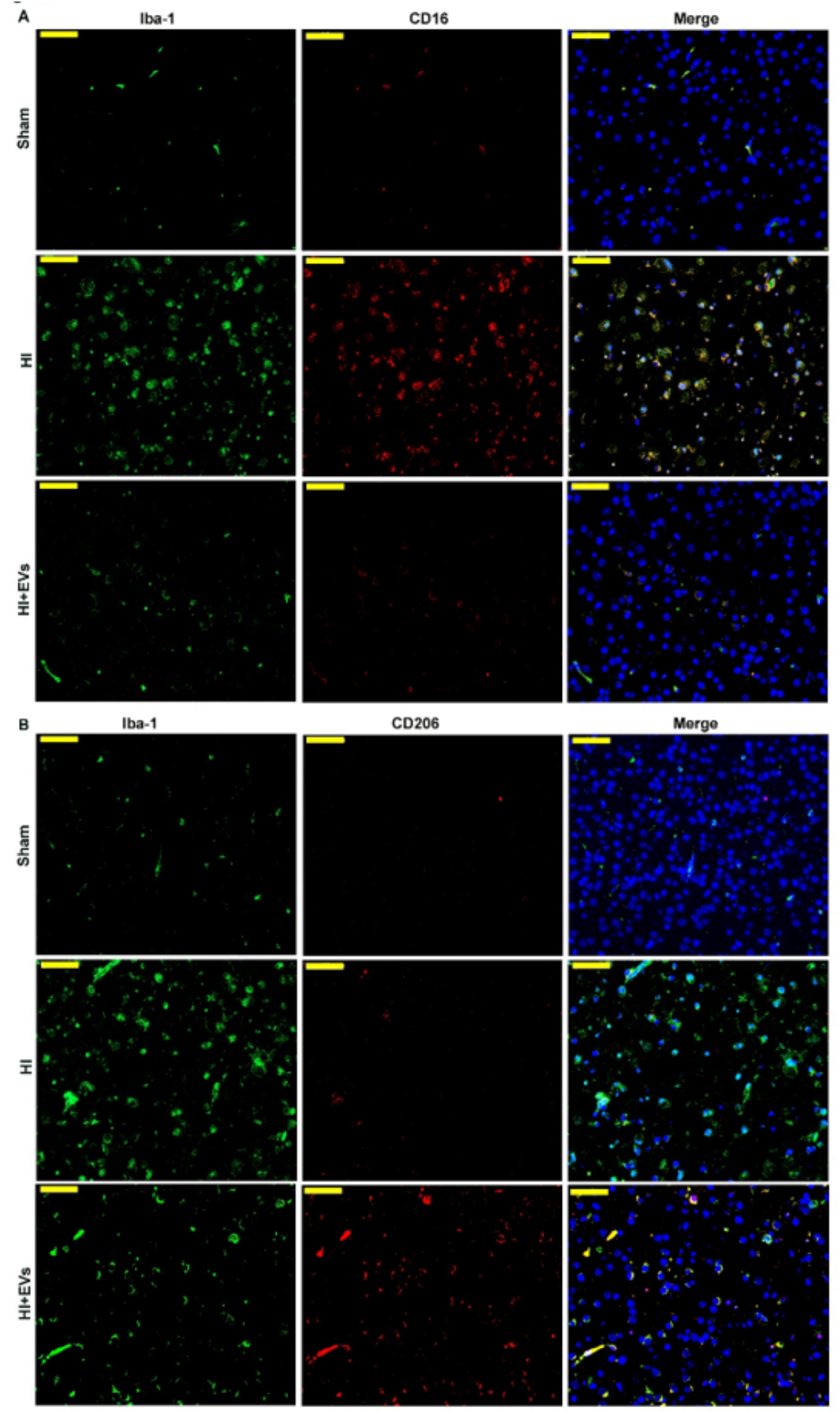

Figure 6

MiR-21a-5p in MSCs-EVs promoted M2 microglia polarization following HI insult (A) Representative photographs of immunofluorescent staining of Iba-1 (green), CD16 (red) within ipsilateral cortex were measured at $72 \mathrm{~h}$ post-HI insult. Scale Bar = $50 \mu \mathrm{m}$. (B) Representative photographs of immunofluorescent staining of Iba-1 (green) and CD206 (red) within ipsilateral cortex were measured at $72 \mathrm{~h}$ post-HI insult. Scale Bar $=50 \mu \mathrm{m} . \mathrm{N}=4$ /group. 


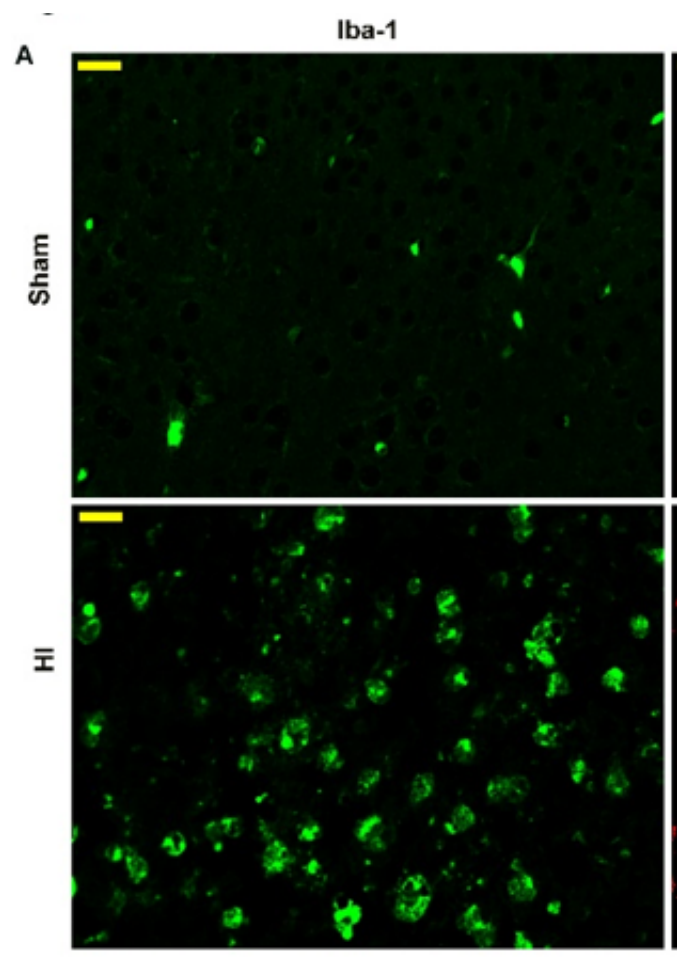

B
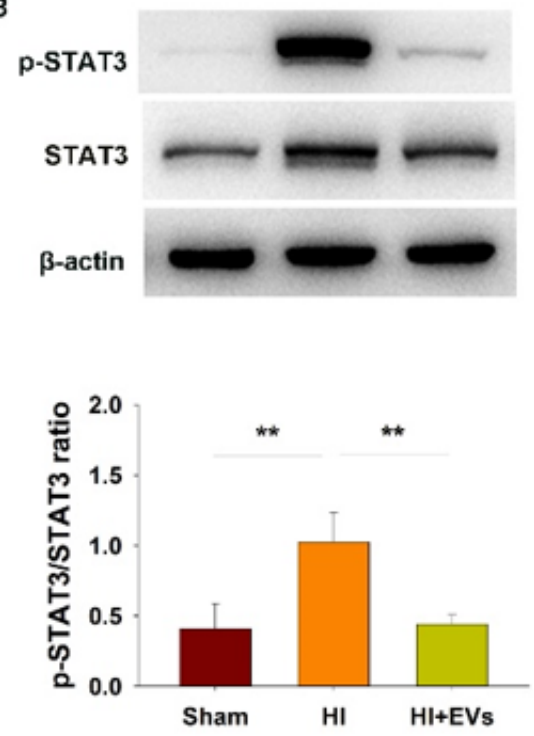

p-STAT3
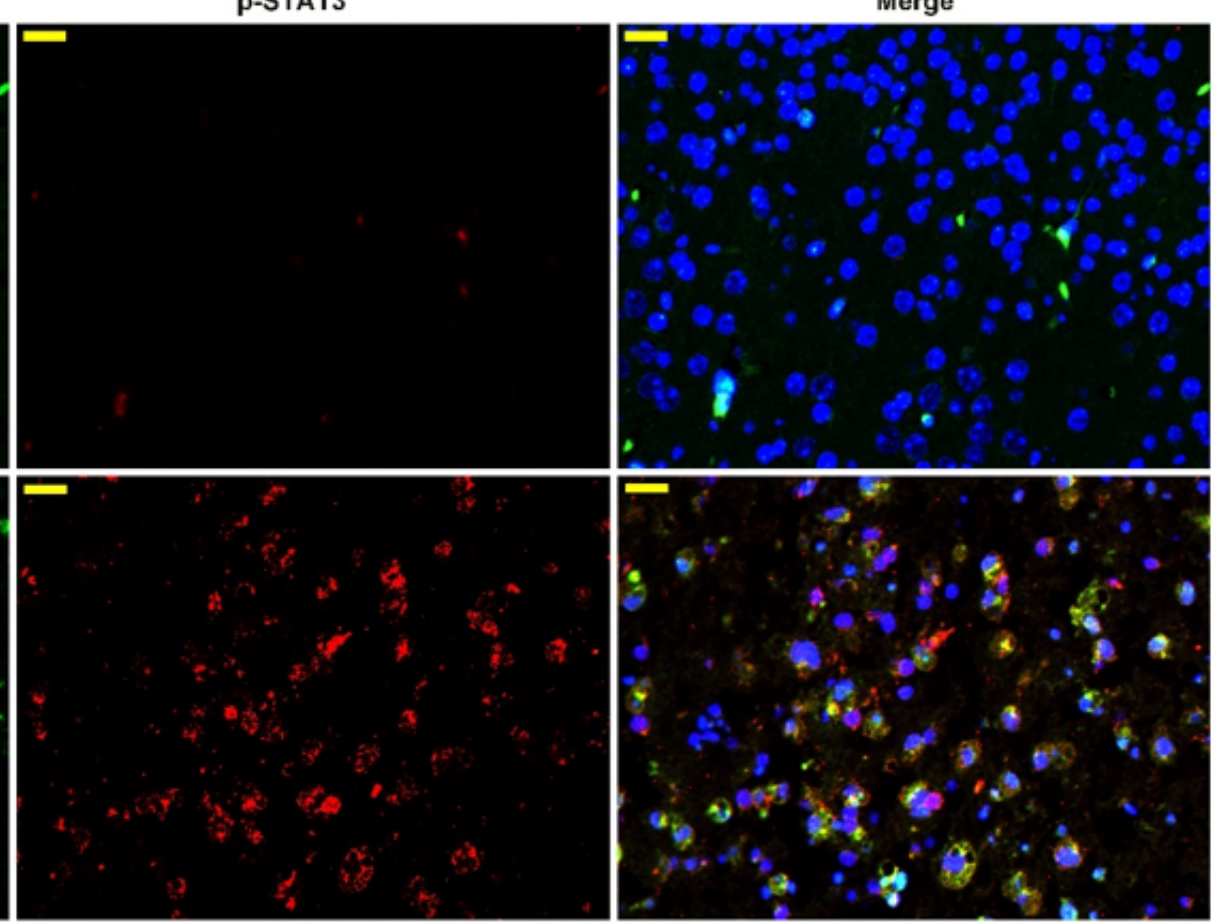

C
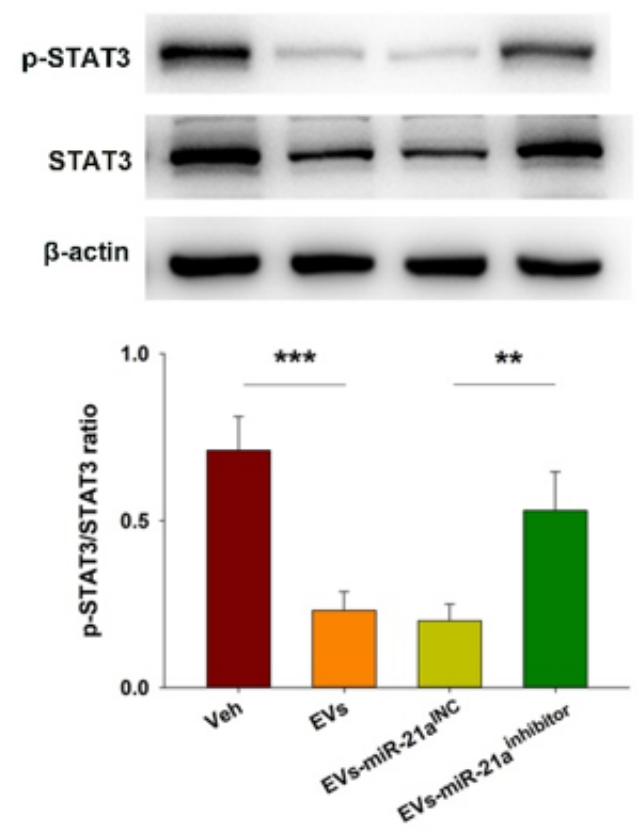

\section{Figure 7}

MiR-21a-5p in MSCs-EVs regulated STAT3 signaling pathway in the right cortex following HI insult (A) Representative photographs of immunofluorescent staining of p-STAT3 (red), Iba-1 (green) within ipsilateral cortex were measured at $72 \mathrm{~h}$ post-HI insult. Scale bar $=50 \mu \mathrm{m}$. (B) The levels of p-STAT3 and STAT3 within ipsilateral cortex were measured in the presence or absence of MSCs-EVs at $72 \mathrm{~h}$ post-HI insult. $\mathrm{N}=4$ /group. (C) The levels of $\mathrm{p}-\mathrm{STAT} 3$ and STAT3 within ipsilateral cortex were measured in the presence or absence of EVs, EVs-miR-21 ainhibitor, EVs-miR-21alNC. $\mathrm{N}=4$ /group. Values represent the mean $\pm S D,{ }^{*} p<0.01,{ }^{\star *} \mathrm{p}<0.001$ according to ANOVA with Bonferroni correction. 


\section{Supplementary Files}

This is a list of supplementary files associated with this preprint. Click to download.

- Supplementarymaterial.docx 